\title{
Clinical and Trichoscopic Correlation of Scalp in Patients Who had undergone Hair Transplantation
}

\author{
${ }^{1} \mathrm{C}$ Madura, ${ }^{2}$ Neelima Ravipati, ${ }^{3} \mathrm{BS}$ Chandrashekar
}

\begin{abstract}
Hair transplantation and dermatoscopy are two growing fields in dermatology. We present clinical and dermascopic evaluation of patients who had undergone hair transplantation in our institute and also in patients who sought for correction for already performed hair transplantation for various reasons. Scalp examination of donor and recipient zones was done using hand held dermatoscope and assessed for aesthetic outcome and complications if any in these zones. The trichoscopic examination helps dermatologist to look into the tiny intricacies and also to correlate these with clinical examination, which helps the hair transplant surgeon to understand the technical mistakes and correct the defects for perfection. From these observations we can conclude that dermascope is an important pocket tool for hair transplant surgeons.
\end{abstract}

Keywords: Donor-recipient area, Hair transplantation, Trichoscopy.

How to cite this article: Madura C, Ravipati N, ChandrashekarBS. Clinical and Trichoscopic Correlation of Scalp in Patients Who had undergone Hair Transplantation. Int J Dermoscop 2017;1(1):20-25.

Source of support: Nil

Conflict of interest: None

\section{INTRODUCTION}

The hair transplantation (HT) and trichoscopic evaluation are two growing fields in dermatology. Dermatoscope is a simple user-friendly tool used extensively by the dermatologist to perform trichoscopy. ${ }^{1}$ It can be considered as stethoscope for an hair transplantat surgeon, as it assesses the scalp and offers both diagnostic and prognostic insights. There are no studies for dermascopic analysis of transplanted scalp in the literature. We present clinical and dermascopic evaluation of the patients who had undergone HT and also in patients who sought for correction of already performed HT for various reasons.

${ }^{1}$ Consultant Dermatologist \& Dermatosurgeon, ${ }^{2}$ Fellow, ${ }^{3}$ Chief Dermatologist

${ }^{1-3}$ Cutis Academy of Cutaneous Sciences, 5/1, 4th main, MRCR layout, Vijayanagar, Bengaluru, India

Corresponding Author: C Madura, Consultant Dermatologist \& Dermatosurgeon, Cutis Academy of Cutaneous Sciences, 5/1 4th main, MRCR layout, Vijayanagar, Bengaluru, India, email: maduradr@gmail.com

\section{CLINICAL AND DERMASCOPIC OBSERVATIONS OF DONOR AND RECIPIENT ZONES}

We examined the scalps of hair transplanted patients from immediate postoperative period. We also examined the patients who presented to us for the first time for hairline corrections (to add more density, laser hair removal of very low-placed hairline and for esthetic complications, such as pits and cobbling). Trichoscan was performed with handheld dermascope of $10 \times$ magnification. The trichoscopic digital imaging (fotofinder 10-160X) system with epiluminescence microscopy with video documentation was also used for the evaluation..$^{2,3}$ Clinical and dermascopic evaluations were done in both donor and recipient zones. The scalp examination in donor zone included mainly the scar assessment and evaluation of posttransplant density. Recipient site was assessed for the density of transplanted hair, angle of exiting hair, angle of the hair in the hairline, and overall esthetic appearance.

\section{DONOR ZONE}

The donor zone scars can be secondary to the follicular unit extraction (FUE) or follicular unit transplantation (FUT). Dermascopic examination of donor zone can be done as early as day 3, it appears as tiny pinkish flares on dermascopy (Fig. 1). It shows decreased density of the harvested area post FUE. Sometimes, we also see sterile folliculitis at the site of emerging hair (Fig. 2). After complete healing, FUE scars appear as white flares (Fig. 3). The size of flares depends on the size of punches used for harvesting the grafts. It is ideal to use 0.8 to $0.9 \mathrm{~mm}$ punches, only to avoid large scars. ${ }^{4}$ Too close harvest can appear as linear scars. Clinically, they have moth-eaten appearance and on trichoscopic examination, these areas appear as linear white flares (Fig. 4).

Clinically, FUT scar can be flat and atrophic or broad and hypertrophic depending on the patient's inherent characteristics of the scalp and also the breadth of the strip harvest. For esthetically better scar, trichophytic closure is always preferred. ${ }^{4}$ Figures $5 \mathrm{~A}$ to $\mathrm{C}$, show wound closure of FUT with and without the trichophytic closure.

\section{Recipient Site Examination}

The recipient site is assessed for density, flow of hairs (angle of hairs), and Scars. Trichoscopy of recipient site 


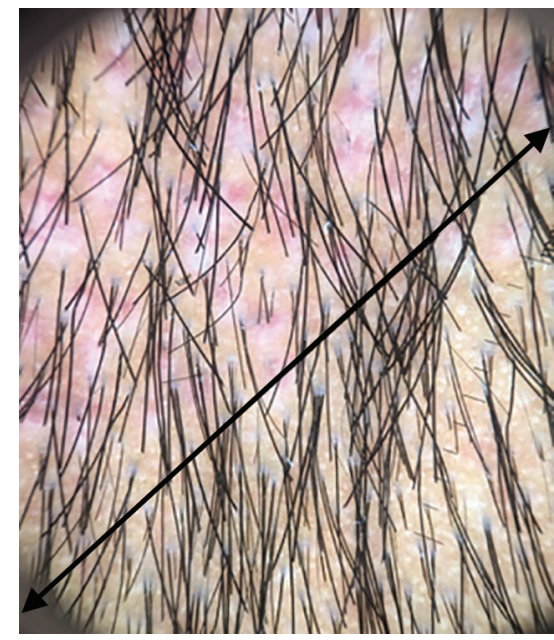

Fig. 1: Postoperative picture of donor zone at day 10. Red flare at the harvest site with decreased density (upper half of the diagonal zone) and nonharvest zones (lower half of the diagonal zone)

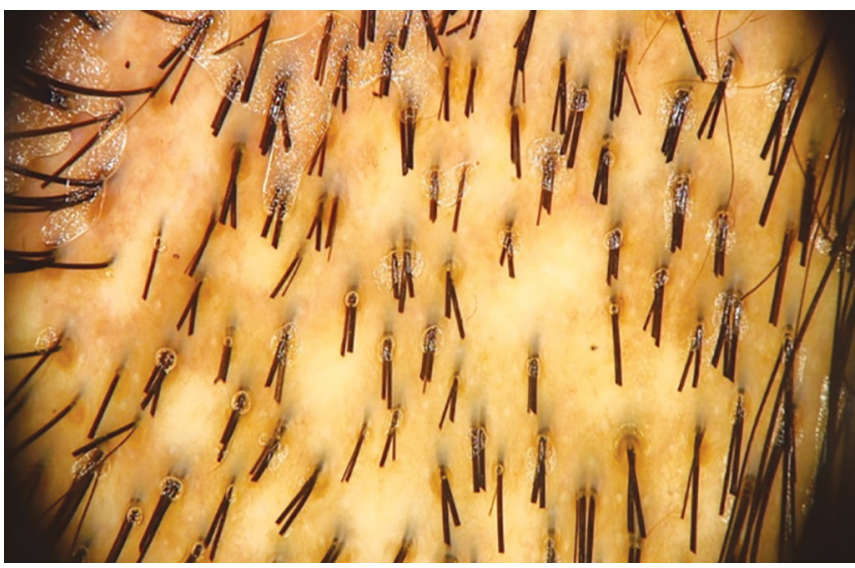

Fig. 3: White flares in the donor zone (healed FUE scars)

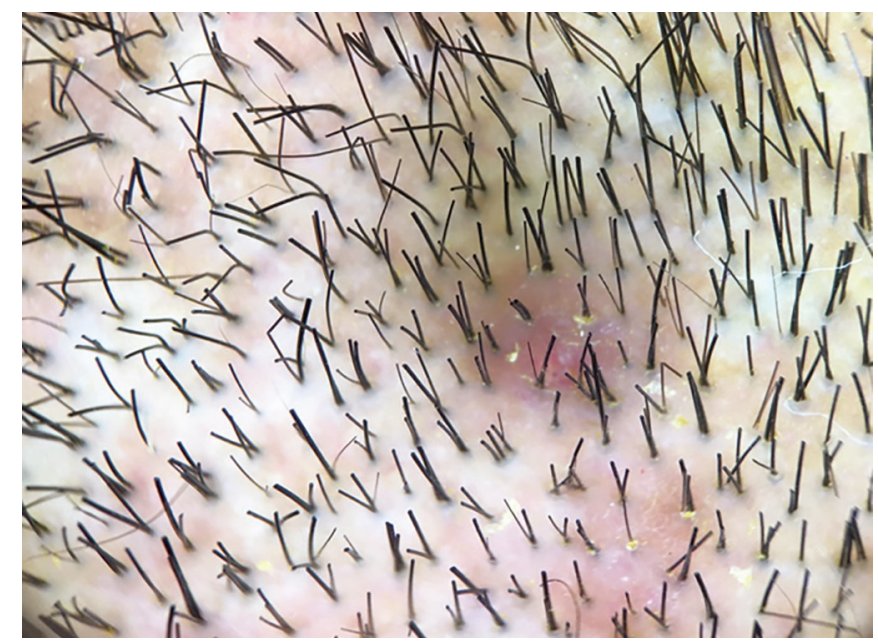

Fig. 2: Donor zone folliculitis

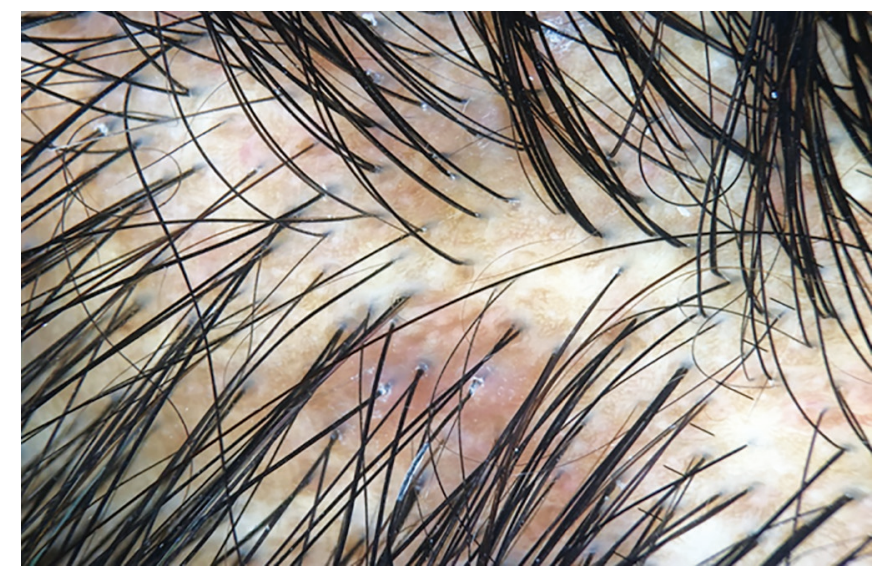

Fig. 4: Linear white flares due to close harvesting of FUE grafts
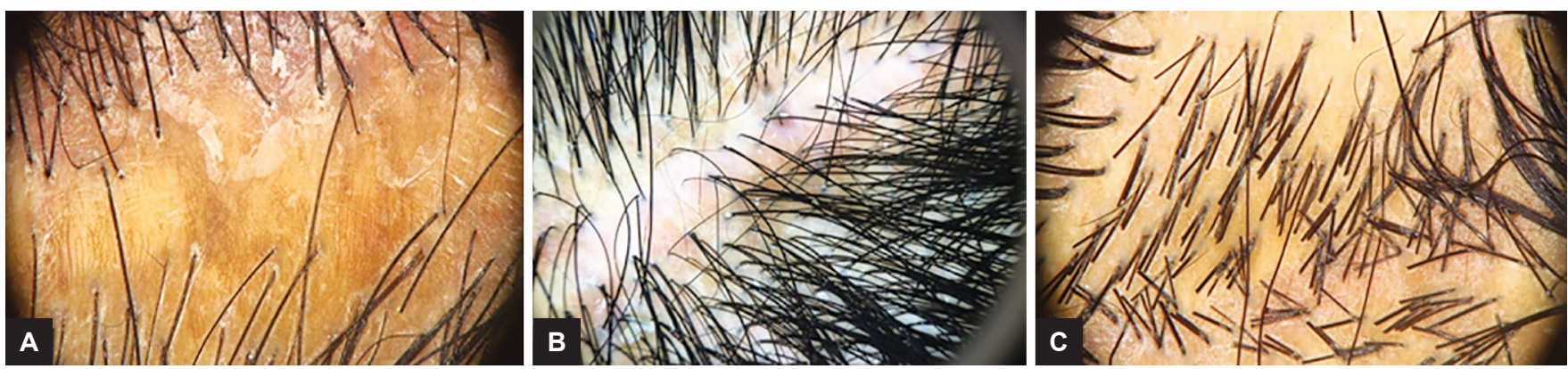

Figs 5A to C: Linear scar secondary to wound closure of FUT: (A) Without trichophytic method; (B) with trichophytic method, with emerging hairs from center of scar; and (C) linear inconspicuous scar secondary to wound closure of FUT with trichophytic method (trimmed area)

on day 3 assesses the early uptake of graft, extent of crusting, and extent of empty slits. The transilluminating light source and magnifying optics of dermascope with both polarized and nonpolarized light give complimentary information of transplanted grafts positioning by nonpolarized light at epidermal level; whereas blood vessels and cicumferential epithelial proliferation looking as red color and white streaks respectively indicate early development and survival of the grafts at dermal level with polarized light. The crust is clinically well evident, Fig. 6A shows the dermascopic appearance of crust on day 3. At the end of 1st week, on dermascopy, we can make out adherent or less adherent crust. The adherent crust indicates improper cleaning of scalp. Figure 6B shows complete crust dislodgement at day 10. Early dermascopic examination also helps to identify the empty slits, where implants are missed, which is clinically not evident (Fig. 6C). Figures 7A and $\mathrm{B}$ show immediate posttransplantation areas at 


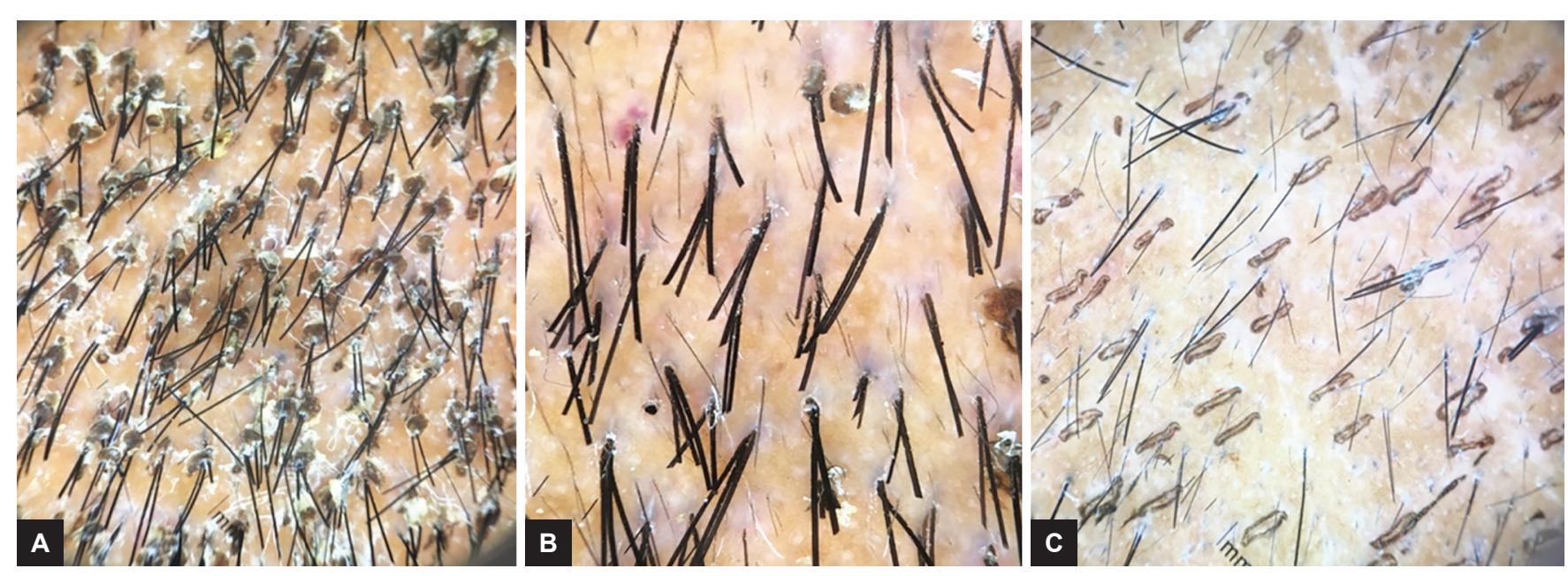

Figs 6A to C: Sequel of healing in recipient zone: (A) At day 3 with crust; (B) day 10 after crust dislodgement; and (C) empty slits indicating missed transplantation areas at day 3
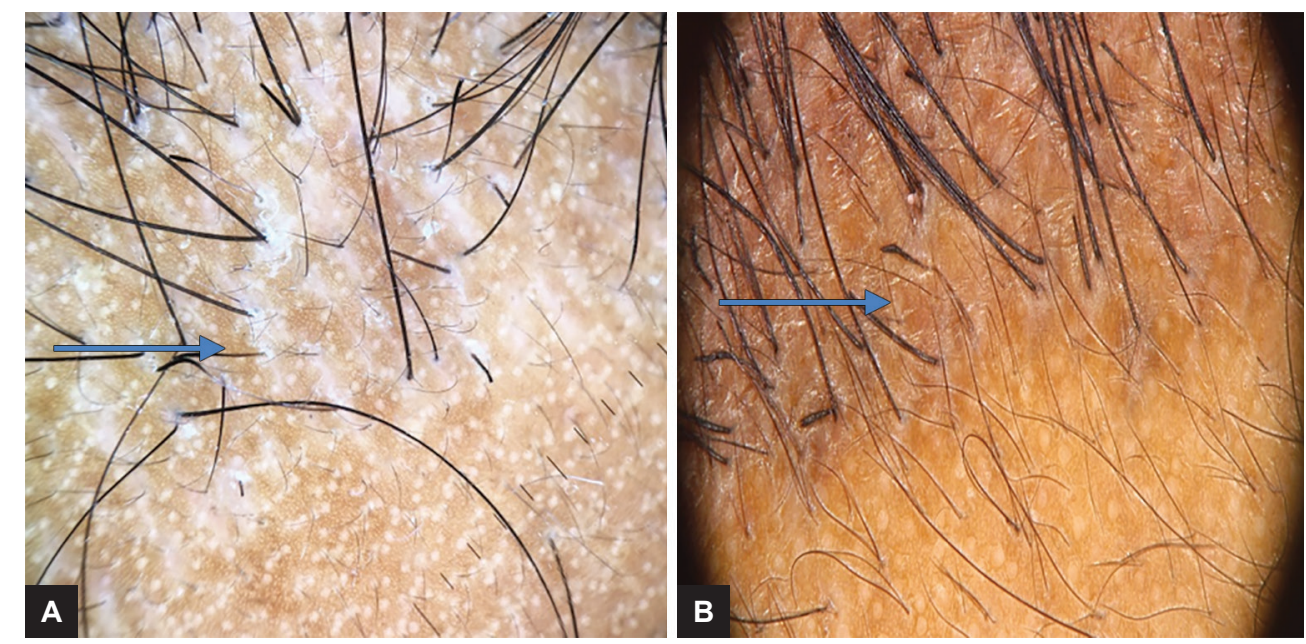

Figs 7A and B: Sequel of healing in recipient zone: (A) Empty areas shows initial hair loss at 6 weeks; and (B) at 3rd month shows regrowth of hairs
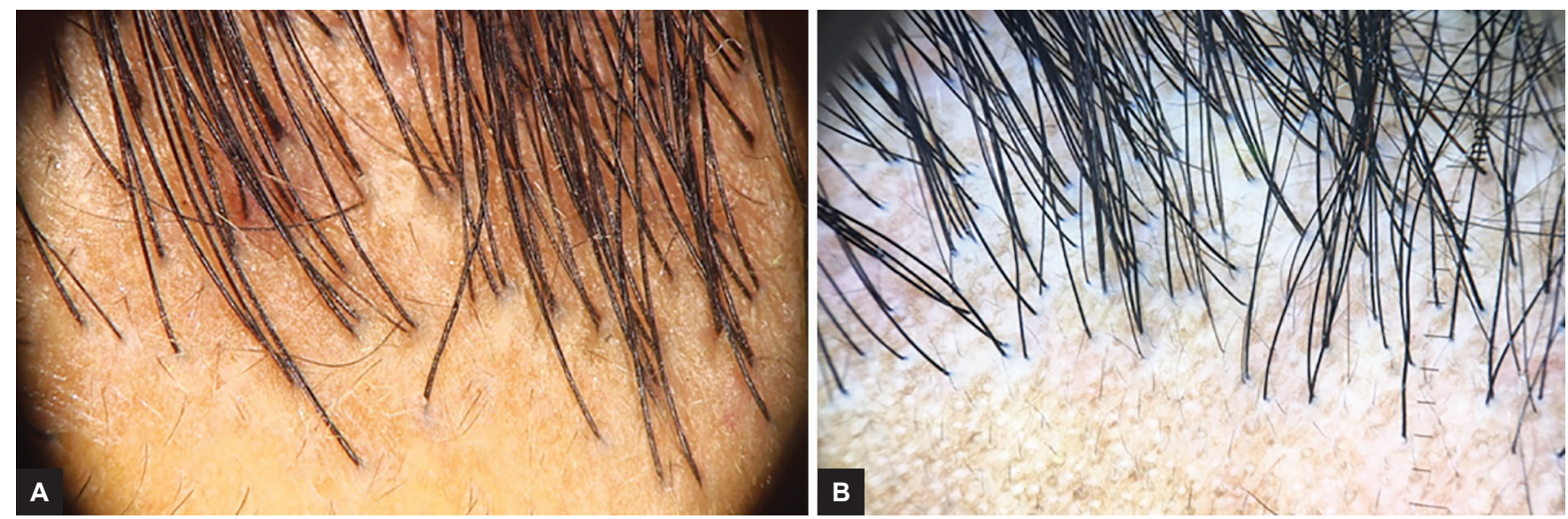

Figs $8 \mathrm{~A}$ and $\mathrm{B}$ : Esthetically good hairline

6 weeks and 3 months. Hairline creation is the signature of each surgeon, it speaks the quality of HT. Clinically, hairline looks soft with singular hairs and good density. There are no mutifollicular units in the hairline. ${ }^{4}$ Figures $8 \mathrm{~A}$ and B show- esthetically pleasing hairline without bulky grafts and curling of hairs. Figures $9 \mathrm{~A}$ and B shows space hair with varied angle and direction. The hair emerging angle is not acute. Figures $10 \mathrm{~A}$ and B show the transplanted hairs in between the existing vellus hair. There is a perfect matching of the angle of emergence of 


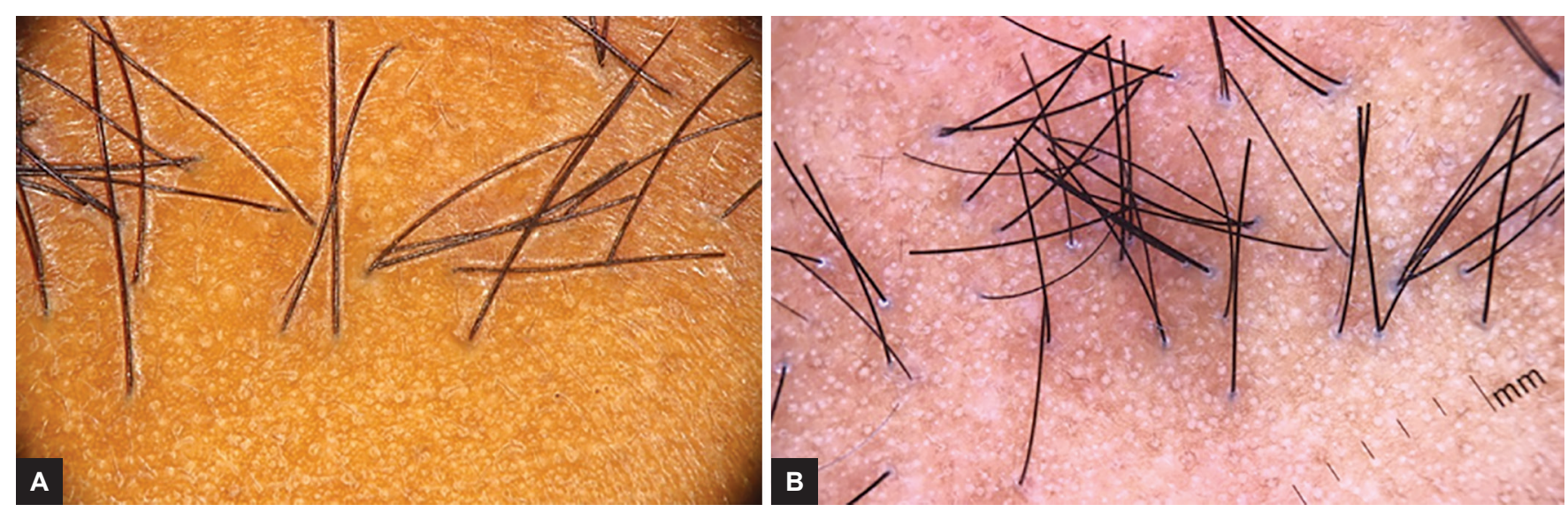

Figs $9 \mathrm{~A}$ and B: Esthetically poor hairline
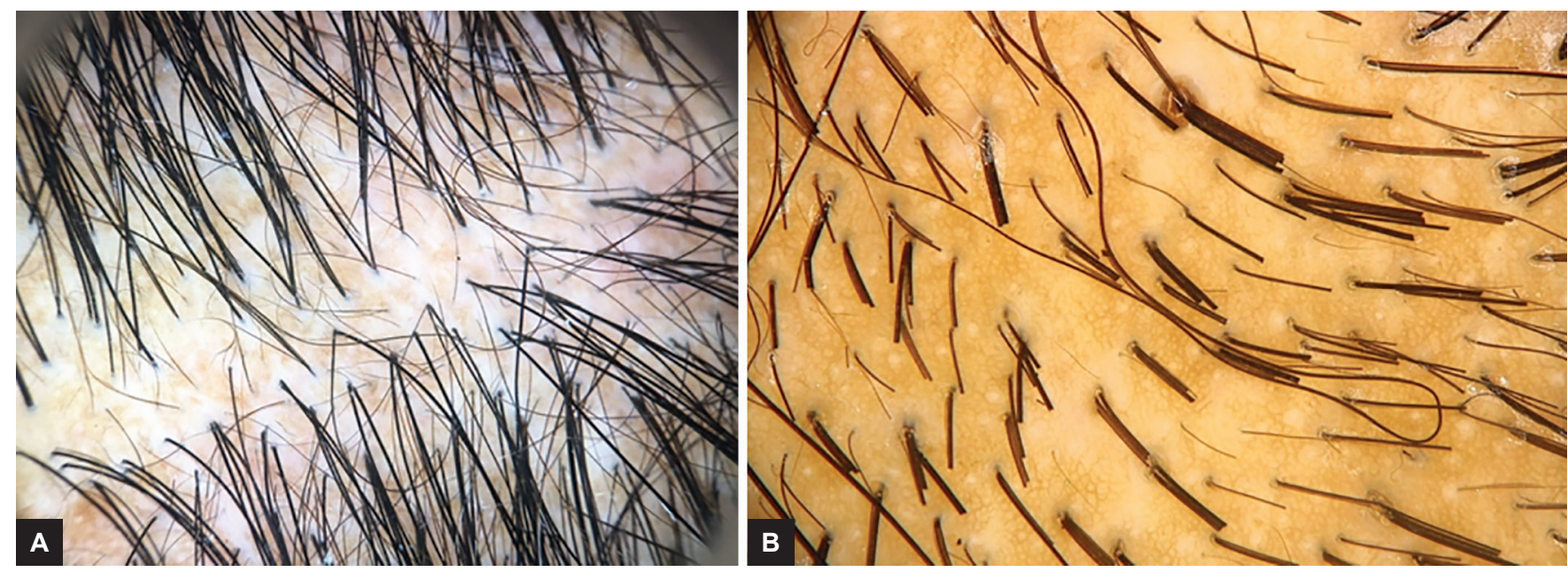

Figs 10A and B: (A) Transplanted hair in between the existing vellus hair; and (B) trimmed hair, angle oftransplanted hairs are same as the existing hairs
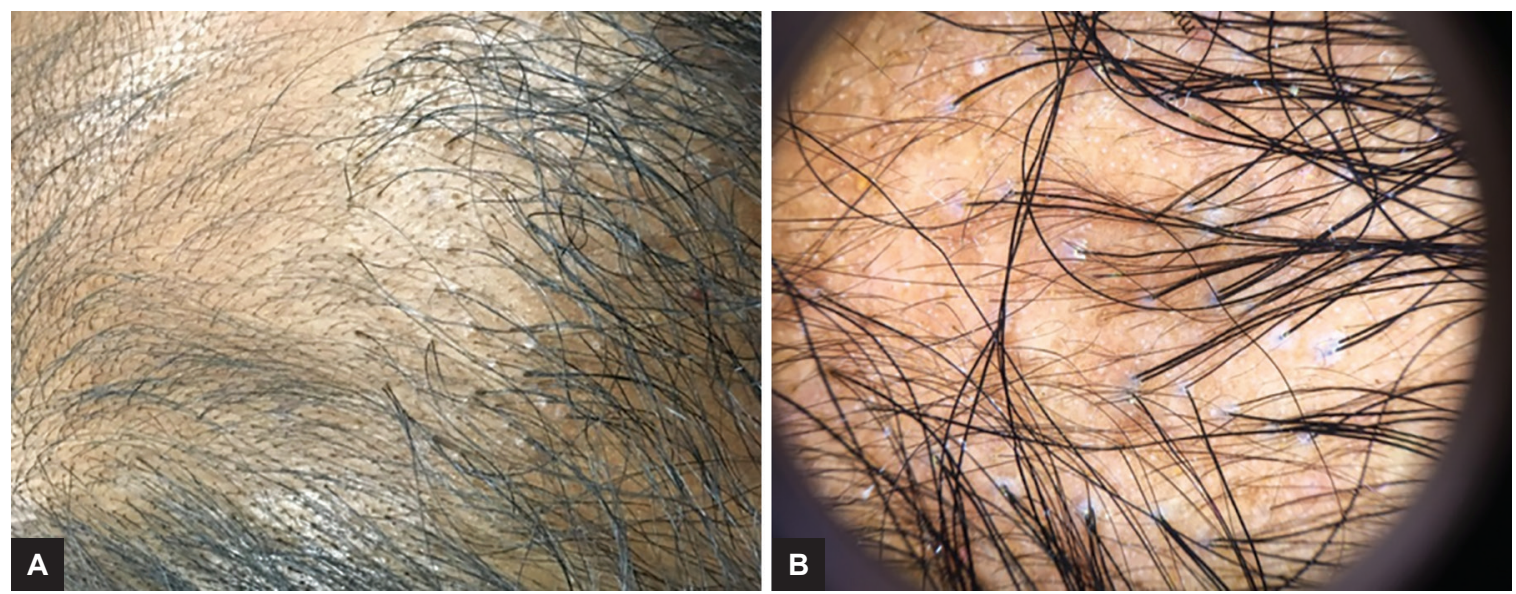

Figs 11A and B: (A) Posttransplant cobble stoning; and (B) tenting with dermascopic image

transplanted hairs with the existing hairs. Esthetic complications, such as pits and cobblestoning are evident in technically poorly performed HT. Cobbling appears as papules around hair follicles and pitting appears as depressed ice-pick like scars around the hair follicle (Figs 11A and B) and (Figs12A and B).
Cobble stone deformity is due to superficial graft placement due to shorter slits. Pitting is due to the placing of grafts in longer slits (longer than the grafts). Figures 13A to $C$ show correlation of normal implanted zone with esthetic complications. On tricoscopy, uncomplicated zone shows grey hue around the follicles. Cobbled 


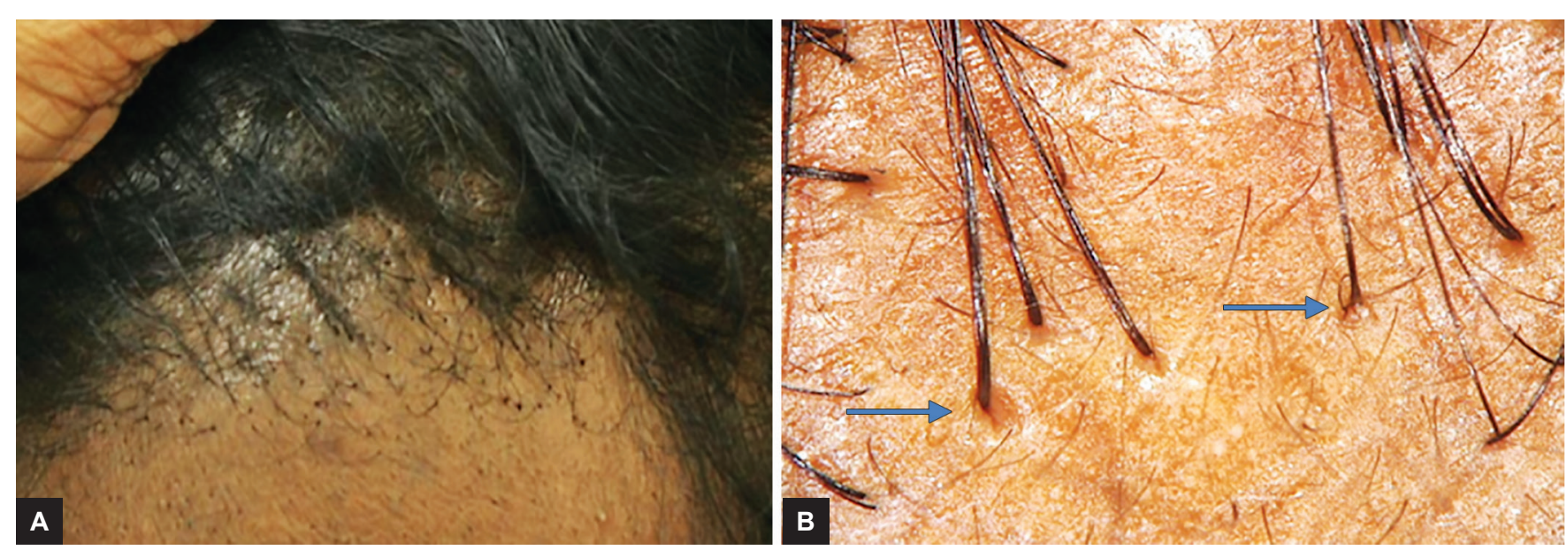

Figs 12A and B: Clinical and dermascopic images of pits on polarized light

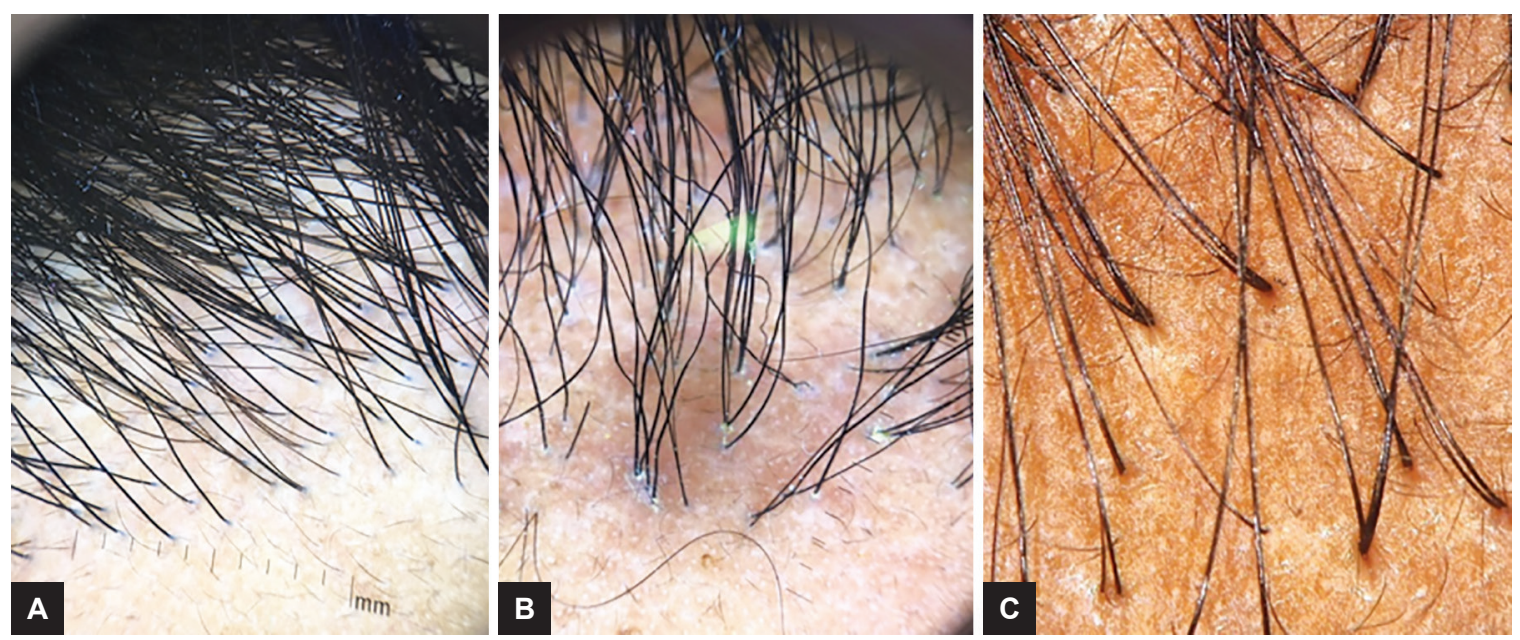

Figs13A to C: (A) Uncomplicated natural hairline. Perfollicular grey hue is present; (B) hairline with cobbling deformity and also wrong angle and multifollicular units in hairline. Cobbling presents as accentuation of grey zone; and $(C)$ pitting

zone shows accentuation of this phenomenon. Pitting is evident in polarized light as valley like margins sloping toward the follicle.

\section{DISCUSSION}

The trichoscope has a lot of applications in HT, it aids accurate diagnosis, assess the donor area and evaluates the results after surgery.

First, the trichoscan defines the candidate for surgery. The diagnosis of androgenic alopecia is straightforward with definitive clinical and dermascopic picture. ${ }^{5}$ The trichoscopy can be important tool to differentiate classical case of androgenic alopecia from diffuse unpatterned androgenic alopecia (DUPA) (diffuse unpatterned androgenic alopecia), an underreported entity. The DUPA poses challenge for hair transplant surgeon. It differs from male patterned hair loss in lacking the stable permanent zone. Diagnosing DUPA is very important because selecting them for HT can prove to be a disaster. ${ }^{6}$ On dermascopy, the occipital region in classic androgenic alopecia and that of normal control does not show any difference in the area. ${ }^{7-9}$ The trichoscan is valuable in differentiating diffuse alopecia areata from alopecia incognito. ${ }^{3}$

Second, the trichoscope assesses the donor zone. The safe donor zone is the horseshoe-shaped area of 5 to $7 \mathrm{~cm}$ width overlying the mid-occipital region. ${ }^{4}$ However, the boundaries of safe zone should be individual to each patient. The trichoscopic evaluation is very valuable here. It helps in assessing the density, grouping so that number of sessions and graphs for a patient can be planned and documented. A donor supply to recipient demand ratio is estimated that helps the physician determine the good/poor candidate for surgery. The digital imaging also assesses terminal vs miniaturized (vellus) hair or the amount of anagen $v s$ telogen hair in an area that can be quantified and compared. ${ }^{10}$ The donor density can be quickly and accurately measured in multiple areas providing an estimate of donor supply. The device assesses the parafollicular area for any disease pathology (seborrhea, dermatitis, psoriasis and healed folliculitis scar). The device not only assesses the density but also the 
emergence of hair and intracutaneous direction of follicle can be assessed.

Third, the trichoscopy documents the hair growth and loss. If early dermascopic examination of scalp shows many empty slits, it infers inefficient filling of the areas. This can be utilized to educate the assistants to correct themselves for better results.

The trichoscopic image of pretransplant area can be compared with image of posttransplant area of single point. This is more important in handling dissatisfied patients. It easily differentiates the thicker transplanted hair growing in the midst of thinner vellus hair (Figs 10A and $\mathrm{B})$. The device also assesses the other features of hair -transplantation, such as angle of hair and esthetic complications (pits and cobbling) (Figs 13A to C).

Fourth, the trichoscan of donor area after HT, especially the FUT scar which is closed by trichophytic method shows regrowth of hair within the scar (Figs 5A to C). These pictures can motivate the patients undergoing HT to accept the FUT technique who have scar phobia.

Therefore, trichoscopic examination helps dermatologist to look into these tiny intricacies and correlate these with clinical examination. This helps them understand the technical mistakes and correct the defects for perfection.

\section{CONCLUSION}

The steps in HT involve assessment, harvest, and transfer of thousands of individual follicular units to the recipient site. This mandates the usage of technology at all points. Hair transplant surgeons run a busy clinic and this user-friendly tool can aid in patient assessment more scientifically. Dermascope, as a diagnostic tool, monitors treatment response and also gives an insight on prognosis. This article would encourage all the readers to take up larger studies to evaluate the transplanted scalp for more growth in this field.

\section{REFERENCES}

1. Tosti A. Hair shaft disorders. In: Tosti A, editor. Dermoscopy of hair and scalp: pathological and clinical correlation. Illustrated edn. USA: CRC Press; 2007. p. 51-53.

2. Rudnicka L, Olszewska M, Rakowska A, Kowalska-Oledzka E, Slowinska M. Trichoscopy: a new method for diagnosing hair loss. J Drugs Dermatol 2008 Jul;7(7):651-654.

3. Tosti A, Duque-Estrada B. Dermoscopy in hair disorders. J Egypt Women Dermatol Soc 2010;7(1):1-4.

4. Harris JA. Follicular unit extraction. In: Unger WP, Shapiro R, Unger R, Unger M, editors. Hair transplantation, 5th edn. London: Informa Healthcare; 2011. p. 291-296.

5. Inui S, Nakajima T, Itami S. Scalp dermoscopy of androgenetic alopecia in Asian people. J Dermatol 2009 Feb;36(2):82-85.

6. Bernstien RM. Follicular unit hair transplantation. In: Robinson JK, Hanke CW, Siegel DM, Fratila A, editors. Surgery of skin, 2nd edn. Mosby: Elsevier; 2010. p. 485-512.

7. HU R, Xu F, Han Y, Sheng Y, Qi S, Miao Y, Yang Q. Trichoscopic findings of androgenetic alopecia and their association with disease severity. J Dermatol 2015 Jun; 42 (6):602-607.

8. Rakowska A,Slowinska M,Kowalska-OledzkaE,Olszewska M, Rudnicka L. Dermoscopy in female androgenic alopecia: method standardization and diagnostic criteria. Int J Trichology 2009 Jul;1(2):123-130.

9. Galliker NA, Trüeb RM. Value of trichoscopy versus trichogram for diagnosis of female androgenetic alopecia. Int J Trichology 2012 Jan;4(1):19-22.

10. Elghblawi E. Trichoscopy simplified. Open Dermatol J 2015 May; 9:12-20. 